\title{
LA TRADICIÓN DE LOS ESTUDIOS AGRARIOS/ RURALES EN LA GEOGRAFÍA ESPAÑOLA: EL CASO DE ARAGÓN *
}

\author{
Ma Luisa FRUTOS MEJÍAS \\ Universidad de Zaragoza
}

La tradición de los estudios sobre Geografía Agraria/Rural en Aragón es precoz, en relación con la incorporación de España a la Geografía moderna. Cuando Casas Torres ocupa la cátedra de la Universidad de Zaragoza en 1944, orienta a una primera generación de alumnos hacia este enfoque moderno de la Geografía, que ya había sido asumido desde hacía más de medio siglo en otros países europeos.

La influencia más directa que se recoge es la del posibilismo francés de base historicista, centrando la atención investigadora en la Geografía Regional, que, obviamente, estaba por hacer en España, teniendo en cuenta ese carácter predominantemente agrario que tuvieron las regiones francesas en las primeras décadas del siglo y que perduraba en España todavía en esas fechas, tal como ya señala Gil Oncina en una síntesis sobre el estado de la cuestión de la investigación geográfica sobre el mundo rural (Aportación al XXVII Congreso de la UGI, 1992).

La propia tesis doctoral de Casas Torres se interesaba ya por el mundo rural, concretamente por la vivienda y el núcleo rural en la huerta valenciana (1943)

Así, las tesis regionales de Alfredo Floristán Samanes, sobre la Ribera Tudelana de Navarra; de Manuel Ferrer Regales, sobre el Campo de Cariñena; de Fernández Marco, sobre el Canal Imperial de Aragón; de García Manrique sobre las comarcas de Borja y Tarazona; de Higueras Arnal sobre el Alto Guadalquivir, dirigidas por Casas y publicadas entre 1950 y 1962 tienen un alto número de páginas dedicado al análisis de la agricultura, la población y el poblamiento rural. En todas ellas se hace un análisis del pasado de la zona, con apoyo de documentos y Amillaramientos antiguos, buscando la explicación evolutiva y del paisaje actual en su historia.

\footnotetext{
* Aportación al XII Coloquio de Geografía Rural ¿Qué futuro para los espacios rurales? León, 15-17 de septiembre, 2004. Mesa redonda: La tradición de los estudios agrarios/rurales en la Geografía Española.
} 
Igualmente, las Tesis de Licenciatura que se publican es ese decenio se estructuran en torno al esquema metodológico "Iniciación a la geografía local" que el propio Casas y sus primeros discípulos redactan y publican en 1953.

Paralelamente, Casas Torres organiza en Zaragoza en el Instituto de Geografía Aplicada Juan Sebastián Elcano, del CSIC, y funda la revista Geográphica, cuyo primer número sale a la luz en 1954. En ella, junto a otros temas geográficos, didácticos y de investigación, se publican desde el principio artículos sobre cuestiones agrarias, 6 en el primer periodo de 1954 a 1962, como recoge Bosque Maurel en el apartado dedicado a Casas y a esta revista (1992), a los que se suman en los años sucesivos otros 40 artículos sobre estas cuestiones.

La temática es, en ellos, monográfica (sectorial): el viñedo, los regadíos...., aunque también incluye el estudio de alguna comarca agraria (Monegros).

La colaboración con el Instituto de Estudios Pirenaicos (hoy Instituto Pirenaico de Ecología), también del C.S.I.C., propició encuentros científicos y la publicación en la revista Pirineos de temas similares a los que se han citado.

Esta etapa, con el método clásico de investigación citado, culmina en el Coloquio sobre Geografia Agraria, organizado en Salamanca en octubre de 1965 y publicado en 1966, en cuyas actas constan comunicaciones de Casas Torres y Mensua Fernández sobre la aplicación de la fotografía aérea a la cartografía de los usos del suelo, de Higueras Arnal sobre una aplicación de estas técnicas, de Ferrer Regales sobre los paisajes y modos de vida agrarios en la comarca del Gran Bilbao y de Floristán sobre los elementos y factores del paisaje rural, sistematización de los conceptos utilizados en este tema.

En suma, las aportaciones que creo que se pueden destacar son, aparte de esos estudios regionales concretos, la sistematización de un método de análisis para las zonas agrarias a partir de los componentes de los paisajes agrarios, y el uso de esa nueva herramienta que es la fotografía aérea.

En relación con la utilización de esta nueva herramienta que supone una modernización de los métodos de expresión gráfica clásicos, tienen gran interés las cartografías de los usos del suelo de varias provincias del valle del Ebro (MENSUA y SolANS). Del mismo modo se incorpora su uso a las sucesivas tesis regionales elaboradas en la segunda mitad de los 60 y los primeros años 70 .

En esta segunda etapa, las Tesis regionales van incorporando nuevas cuestiones, sin perder el enfoque agrario/rural, de la mano de Frutos, Chueca, Calvo Palacios, García Ruíz, Valenzuela, Rubio, Lasanta y otros (El campo de Zaragoza, Sierras y Valles al NW del Jalón, los Cameros, Población y recursos de la provincia de Zaragoza, Modos y Vidas del Prepirineo oscense, los usos del sue- 
lo en el Pirineo, el Valle del Jalón o los cambios de uso y abandono de tierras labradas en el Pirineo aragonés). Se tienen en cuenta cuestiones económicas y comerciales y problemas locales. Estos nuevos aspectos se reflejan, así mismo, en comunicaciones a congresos, centrando la atención en algún cultivo específico, como la remolacha, los cultivos forrajeros o los frutales, y aunque con un número escaso, también sobre la ganadería aragonesa. Poco a poco se va perdiendo la perspectiva histórica al incidir más sobre los problemas actuales.

Las nuevas orientaciones epistemológicas y metodológicas del neopositivismo y el cuantitativismo marginan en cierto modo los trabajos de Geografia Rural en Aragón (ganan terreno los trabajos sobre Población, Geografía Urbana, industria, turismo...y especialmente Geografía Física), aunque no se abandonan: queda patente en la participación en los Seminarios que organiza la Fundación March sobre paisajes agrarios, con estudios sobre cambios en las estructuras agrarias, en el régimen de tenencias o en los cultivos, e igualmente en la participación en Congresos Nacionales e Internacionales. Pero los métodos siguen siendo similares, si bien se incorpora el manejo de una nueva fuente muy útil: los Censo Agrarios.

La incorporación de los trabajos de Geografía Agraria/Rural a nuevas técnicas, dentro de la concepción cuantitativista, dará lugar a algunos artículos (tímida aportación en Los desequilibrios provinciales en España a través de la rentabilidad agraria provincial) y nuevas orientaciones temáticas, con el uso de la teledetección y la cartografía automática (décadas 80-90) para analizar los bosques aragoneses desde la doble perspectiva ecológica y humanizada (Querol, de la Riva), así como las características, evolución y vigencia de explotaciones agropecuarias tradicionales, como el "mas" de Teruel (Ruíz Budría).

En estas últimas décadas, tras nuestra incorporación a la U.E., aunque los temas estrictamente agrarios siguen siendo objeto de estudio (especialmente los cambios en el sistema agrario) han quedado relegados a un segundo plano por el interés despertado por los procesos de desarrollo rural, que incorporan con fuerza nuevos temas, como la industrialización o el turismo en el medio rural, y por los trabajos aplicados sobre planes parciales de ordenación rural, que tiene en cuenta nuevos conceptos, así como el trabajo interdisciplinar y permiten volver los ojos de nuevo a la idea inicial que dio vida a la Geografía en la Universidad de Zaragoza: su aplicación. Varias aportaciones a Coloquios y Congresos dan fe de este interés, así como los proyectos de Investigación en marcha, financiados por el Ministerio de Ciencia y Tecnología o por la DGA.

A esto responde el Curso de Posgrado "Gestor de Desarrollo Rural", con sede en Huesca, pero dirigido por miembros de nuestro Departamento, que ha propi- 
ciado que algunos geógrafos obtengan un puesto de trabajo en los LEADER o en Ayuntamientos.

Algunas Tesis se orientan específicamente al análisis de la situación y las propuestas de estrategias de desarrollo, como la de Rubio Terrada sobre el Jiloca.

Otro aspecto a destacar en las investigaciones actuales de los geógrafos aragoneses es el acercamiento de los geógrafos físicos a los temas agrarios, en un sentido amplio. Es sobre todo en el estudio del clima donde queda más claro, con trabajos sobre riesgos agroclimáticos, sobre todo heladas y sequías, línea de trabajo que inició Yetano al orientar así la tesis de M.L. Hernandez sobre los riesgos de las heladas en los frutales del Valle del Ebro y ha continuado Cuadrat con trabajos propios, proyectos y dirección de Tesis. En menor medida temas como la erosión de campos abandonados, los cambios de paisaje, las propuestas para la recuperación de pastos o los riesgos de incendio son también puntos de convergencia entre agraristas, geomorfólogos y biogeógrafos.

Finalmente puede añadirse que la estrecha relación que existe con algunas de las Universidades de América Latina ha permitido a algunos de los geógrafos de estos países hacer sus Tesis Doctorales entre nosotros y varias de ellas tienen una clara orientación agraria, con interés creciente por cuestiones de sostenibilidad, agricultura y medioambiente. 